\title{
Computing Technology of a Method of Control Volume for Obtaining of the Approximate Analytical Solution to One-Dimensional Convection-Diffusion Problems
}

\author{
Dalabaev Umurdin \\ Department of Mathematics modeling and informatics, University of World Economy and Diplomacy, Tashkent, Uzbekistan \\ Email: udalabaev@mail.ru
}

How to cite this paper: Umurdin, D. (2018) Computing Technology of a Method of Control Volume for Obtaining of the Approximate Analytical Solution to OneDimensional Convection-Diffusion Problems. Open Access Library Journal, 5: e4962. https://doi.org/10.4236/oalib.1104962

Received: October 8, 2018

Accepted: November 6, 2018

Published: November 9, 2018

Copyright $(9) 2018$ by author and Open Access Library Inc.

This work is licensed under the Creative Commons Attribution International License (CC BY 4.0).

http://creativecommons.org/licenses/by/4.0/

\begin{abstract}
The solution of problem convection-diffusion equation by way of control volume method is considered. Approximate solution of problem is received. Three point scheme of high resolution is constructed.
\end{abstract}

\section{Subject Areas}

Computational Physics

\section{Keywords}

Convection-Diffusion Equation, Control Volume Method, Moved Node

\section{Introduction}

Many physical problems involve the combination of convective and diffusive processes. Convection-diffusion problems arise frequently in many areas of applied sciences and engineering. They occur in fields where mathematical modeling is important such as physics, engineering and particularly in fluid dynamics and transport problems.

Mathematical models of physical, chemical, biological and environmental phenomena are governed by various forms of differential equations.

Convection-diffusion problems are governed by typical mathematical models, which are common in fluid and gas dynamics. Heat and mass transfer is conducted not only via diffusion, but appears due to motion of a medium, too.

When velocity is higher, that is, flow term is larger, a simple convectiondiffusion problem is converted to convection-dominated diffusion problem 
because Peclet number is greater than two. A Peclet number is a dimensionless number relevant in the study of transport phenomena in fluid flows. It is defined to be the ratio of the rate of advection of a physical quantity by the flow to the rate of diffusion of the same quantity driven by an appropriate gradient. For diffusion of heat (thermal diffusion), Peclet number is defined as, $\mathrm{Pe}=\mathrm{F} / \mathrm{D}$ where $\mathrm{F}$ is the flow term and $\mathrm{D}$ is the diffusion term.

Finite volume methods are widely used in computational fluid dynamics. The elementary finite volume method uses a cell-centered mesh and finite-difference approximations of first order derivatives. This paper shows how the finite volume method is applied to a simple model of convective transport: the onedimensional convection-diffusion equation.

There are two primary goals of this paper. The first is to apply the finite volume method to obtain approximately analytical solution. The second one is construct scheme which works for all mesh Peclet number. Readers interested in additional details, including application to the Navier-Stokes equations, should consult the classic text by Patankar [1]. The basic attention at a numerical solution is given to problems of approximation of convective terms [2] [3] [4] [5].

Usually, solution of differential equations by numerical methods is obtained in the form of numbers. Here we will show a possibility of deriving of a solution of differential equations by control volume methods in the approximately-analytical form by the so-called moved node.

Such type research it is considered in work [6] with the finite deference method.

\section{Deriving of the Approximately-Analytical Solution}

Let's consider one-dimensional the convection-diffusion equation on a interval $[W, E]$ :

$$
\frac{\mathrm{d}}{\mathrm{d} x}(\rho u \Phi)=\frac{\mathrm{d}}{\mathrm{d} x}\left(\Gamma \frac{\mathrm{d} \Phi}{\mathrm{d} x}\right)+S(x)
$$

with boundary conditions

$$
\mathrm{d} \Phi(W) / \mathrm{d} x=a_{0} \Phi(W)+a_{1}, \quad \mathrm{~d} \Phi(E) / \mathrm{d} x=b_{0} \Phi(E)+b_{1}
$$

where $u$ a stream velocity in a $x$ direction, $\rho$ a stream denseness, $\Gamma-$ a diffusivity, $S(x)$ - a given function (source), $\Phi$ - unknown function. From an equation of continuity implies that $F=\rho u=$ const.

Let's consider the Equation (1) on segments $[W, E]$. For deriving of the approached analytical solution of a problem by the method of control volume we take an arbitrary point $x \in[W, E]$ and control volume $[w, e]$. In Figure $1, x$ is arbitrary point in control volume. $x$ is so-called moved node.

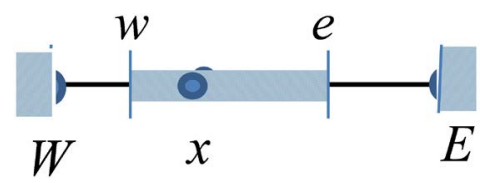

Figure 1. Control volume. 
Let's suppose that, the edge $W$ is arranged in the middle between points $W$ and $x$, and an edge $e-$ in the middle between points $x$ and $E$. Integrating the Equation (1) on control volume and substituting, derivatives the upwind scheme we will receive zero approach

$$
\left(a_{E}+a_{W}\right) \Phi^{0}=a_{E} \Phi_{E}^{0}+a_{W} \Phi_{W}^{0}+\frac{E-W}{2} \cdot S(x)
$$

here $a_{E}=\frac{\Gamma}{E-x}+\max (-F, 0) ; a_{W}=\frac{\Gamma}{x-W}+\max (F, 0)$. As $x \in[W, E]$ an arbitrary point from (3) we can define $\Phi^{0}$ and receive the approached analytical solution of a problem (1) $\left(\Phi_{W}^{0}, \Phi_{E}^{0}\right)$ are defined on a basis (2)). The source term is obtained by assuming that $S$ has the uniform value of the control volume.

To improve approximate solution we take additional grids:

$x_{1}=\frac{x+W}{2}, x_{2}=\frac{x+E}{2}$. Let's write the upwind scheme of type (3) for a segment $[W, x],\left[x_{1}, x_{2}\right]$ and $[x, E]$. Let's receive system from three equations. We exclude the received system, $\Phi^{1}\left(x_{1}\right), \Phi^{1}\left(x_{2}\right)$ and as a result we will receive the improved scheme:

$$
\begin{aligned}
& {\left[\frac{\beta_{1}^{+}}{1+\tau_{1}}+\frac{\alpha_{1}^{-}}{1+\gamma_{1}}\right] \Phi^{1}=\frac{\beta_{1}^{+}}{1+\tau_{1}} \Phi_{W}^{1}+\frac{\alpha_{1}^{-}}{1+\gamma_{1}} \Phi_{E}^{1}+\frac{E-W}{4} \cdot S(x)} \\
& +\frac{1}{1+\tau_{1}} \cdot \frac{x-W}{2} \cdot S\left(W+\frac{x-W}{2}\right)+\frac{1}{1+\gamma_{1}} \cdot \frac{E-x}{2} \cdot S\left(x+\frac{E-x}{2}\right),
\end{aligned}
$$

where $\tau_{1}=\beta_{1}^{-} / \beta_{1}^{+}, \gamma_{1}=\alpha_{1}^{+} / \alpha_{1}^{-}, \beta_{1}^{-}=2 D_{W}+F^{-}, \beta_{1}^{+}=2 D_{W}+F^{+}$, $\alpha_{1}^{-}=2 D_{E}+F^{-}, \alpha_{1}^{+}=2 D_{E}+F^{+}, D_{E}=\Gamma /(E-x), D_{W}=\Gamma /(x-W)$, $F^{-}=\max (-F, 0), F^{+}=\max (F, 0)$. In (4) $\Phi^{1}$ improved value of unknown function in a point $X\left(\Phi_{W}^{1} \equiv \Phi_{W}, \Phi_{E}^{1} \equiv \Phi_{E}\right),\left(\Phi_{W}^{1}, \Phi_{E}^{1}\right.$ are defined on the basis of (2)).

Solving (4) rather $\Phi^{1}$ again for solution improving we will arrive similarly: write the scheme (4) for a segment $[W, x],\left[x_{1}, x_{2}\right]$ and $[x, E]$, and receive the improved analytical solution. Also we will exclude unknowns in points $x_{1}$ and, $x_{2}$ etc. Continuing this process we will obtain

$$
\begin{aligned}
& {\left[\frac{\left(1-\tau_{k}\right) \beta_{k}^{+}}{1-\tau_{k}^{2^{k}}}+\frac{\left(1-\gamma_{k}\right) \alpha_{k}^{-}}{1-\gamma_{k}^{2^{k}}}\right] \Phi^{k}} \\
& =\frac{\left(1-\tau_{k}\right) \beta_{k}^{+}}{1-\tau_{k}^{2^{k}}} \Phi_{W}^{k}+\frac{\left(1-\gamma_{k}\right) \alpha_{k}^{-}}{1-\gamma_{k}^{2^{k}}} \Phi_{E}^{k}+\frac{E-W}{2^{k+1}} \cdot S(x) \\
& \quad+\frac{1-\tau_{k}}{1-\tau_{k}^{2^{k}}} \cdot \frac{x-W}{2^{k}} \cdot \sum_{j=1}^{2^{k}-1} \sum_{i=1}^{j} \tau_{k}^{i-1} S\left(W+j \frac{x-W}{2^{k}}\right) \\
& \quad+\frac{1-\gamma_{k}}{1-\gamma_{k}^{2^{k}}} \cdot \frac{E-x}{2^{k}} \cdot \sum_{j=1}^{2^{k}-1} \sum_{i=1}^{j} \gamma_{k}^{i-1} S\left(x+\left(2^{k}-j\right) \frac{E-x}{2}\right),
\end{aligned}
$$

where $\tau_{k}=\beta_{k}^{-} / \beta_{k}^{+}, \gamma_{k}=\alpha_{k}^{+} / \alpha_{k}^{-}, \beta_{k}^{-}=2^{k} D_{W}+F^{-}, \beta_{k}^{+}=2^{k} D_{W}+F^{+}$, $\alpha_{k}^{-}=2^{k} D_{E}+F^{-}, \alpha_{k}^{+}=2^{k} D_{E}+F^{+}$.

In (5) $\Phi^{k}$ improved value of unknown function in a point 
$x\left(\Phi_{W}^{k} \equiv \Phi_{W}, \Phi_{E}^{k} \equiv \Phi_{E}\right)$. Solving the Equation (5) rather we will receive the approached analytical solution of an initial problem.

\section{Examples}

On Figure 2, solutions of a problem: $50 \Phi^{\prime}(x)=\Phi^{\prime \prime}(x)+50 \sin (\pi x)$ are reduced on segments $[0,1]$ with boundary conditions $\Phi_{W}=0, \Phi_{E}=0$. From the graph it is visible that, in process of magnification to approximate solutions comes nearer to the exact.

On Figure 3, solutions of a problem $5 \Phi^{\prime}(x)=\Phi^{\prime \prime}(x)$ are reduced on segments $[0,1]$ with boundary conditions $\Phi_{W}=0, \Phi^{\prime}(E)=0.5 \Phi_{E}+10$.

\section{Numerical Experiments}

The analytical scheme (5) allows not only receiving the approached analytical solution, but also gives the chance in creation of the qualitative scheme.

Comparisons of exact and difference solutions are on Figure 4 reduced.

Solution of problem $50 \Phi^{\prime}(x)=\Phi^{\prime \prime}(x)+S(x)$ are on Figure 4 graphs on $[0,1]$ with boundary conditions $\Phi_{W}=0, \Phi_{E}=1$. Source $S(x)=10-50 x$ at $x<0.3, S(x)=50 x-20$ at $0.3<x<0.4$ and $S(x)=0$ at $0.4 \leq x$.

In Figure 4, numerical results are received at $h=0.1$ and $P_{h}=5$, (mesh

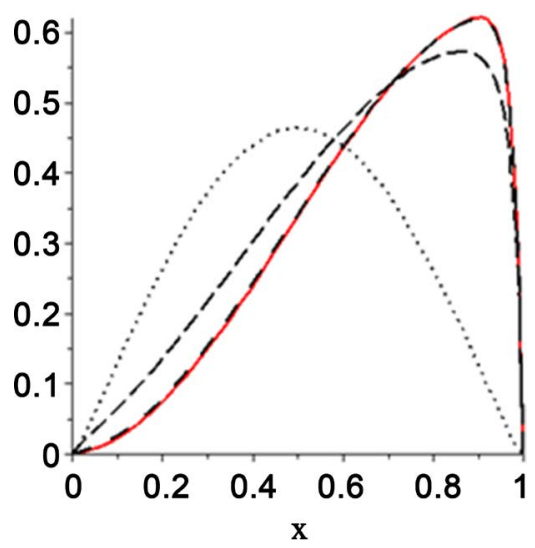

Figure 2. Continuous line-exact, pointwise $-k=0$, dotted $-k=2$, rare dashed $-k=6$.

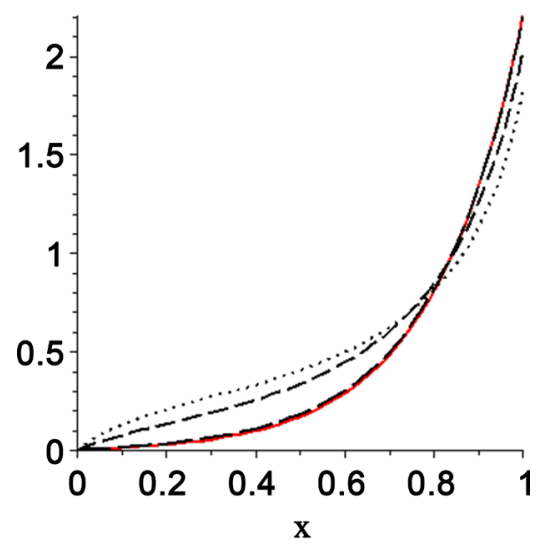

Figure 3. Continuous line - exact, pointwise $-k=0$, dotted $-l=1$, long dashed $-k=6$. 


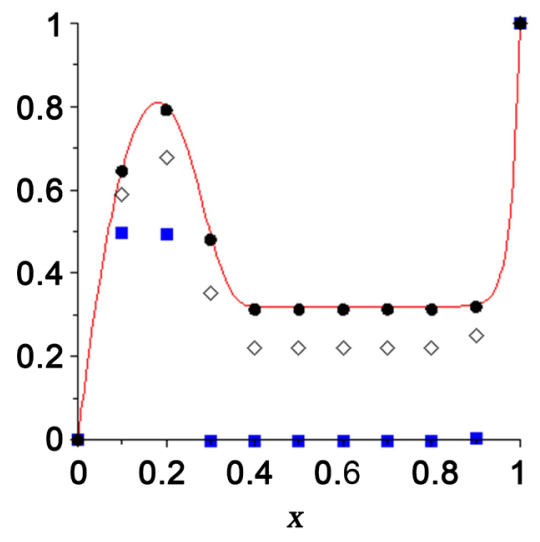

Figure 4. Comparison of various schemes.

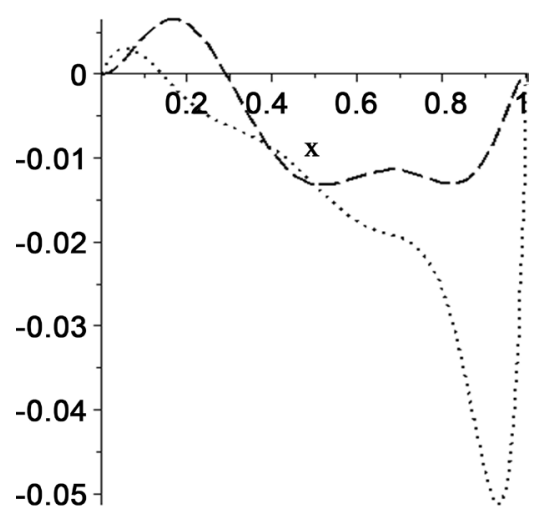

Figure 5. Comparison finite difference and control volume method.

Peclet number). Solid line is exact solutions of the problem. Rectangles under the scheme of Patankar, diamond at $k=2$ and circles at $k=6$. From Figure 4 appears that, the offered schemes yield good results.

Comparison of analytical solutions received finite difference [6] and a method of control volume shows advantage of a method of control volume (Figure 5). On Figure 5, it is shown errors received by these methods. On Figure 5 point wise line corresponds to the difference exact and solutions received by the finite difference method, and dashed-difference exact and solutions of the control volume $(k=4) \quad\left(10 \Phi^{\prime}(x)=\Phi^{\prime \prime}(x)+10 \cos (10 x), \Phi(0)=0, \Phi(1)=1\right)$.

\section{Conclusion}

The approached analytical solution to one-dimensional convection-diffusion problems is received. The algorithm of deriving of a solution is based on the control volume method. The analytical solution allows also constructing the compact scheme. It is the three point scheme of the high order of resolution. It is the scheme that is suitable for any Peclet numbers.

\section{Conflicts of Interest}

The author declares no conflicts of interest regarding the publication of this paper. 


\section{References}

[1] Patankar, S.V. (1980) Numerical Heat Transfer and Fluid Flows. Hemisphere Publishing Corporation, New York. https://doi.org/10.1201/9781482234213

[2] Leonard, B.P. (1990) A Stable and Accurate Convective Modelling Procedure Based on Quadratic Upstream Interpolation. Computer Methods in Applied Mechanics and Engineering, 19, 59-98. https://doi.org/10.1016/0045-7825(79)90034-3

[3] Leonard, B.P. and Mokhtari, S. (1990) Beyond First-Order Upwinding: The Ultra Sharp Alternative for Non-Oscillatory Steady-State Simulation of Convection. International Journal for Numerical Methods in Engineering, 30, 729. https://doi.org/10.1002/nme.1620300412

[4] Leonard, B.P. (1991) The ULTIMATE Conservative Difference Scheme Applied to Unsteady One-Dimensional Advection. Computer Methods in Applied Mechanics and Engineering, 88, 17-74. https://doi.org/10.1016/0045-7825(91)90232-U

[5] Ferreira, V.G., de Queiroz, R.A.B., Lima, G.A.B., Cuenca, R.G., Oishi, C.M., Azevedo, J.L.F. and McKee, S., (2012) A Bounded Upwinding Scheme for Computing Convection-Dominated Transport Problems. Computers and Fluids, 57, 208-224. https://doi.org/10.1016/j.compfluid.2011.12.021

[6] Dalabaev, U. (2016) Difference-Analytical Method of the One-Dimensional Convection-Diffusion Equation. IJISET-International Journal of Innovative Science, Engineering \& Technology, 3, 234-239. http://www.ijiset.com/ 